\title{
La synonymie du point de vue de la pensée linguistique de Coseriu : Étude sur un corpus slovène
}

\author{
Marina Zorman (Ljubljana)
}

\begin{abstract}
Synonymy is a subject of research in various subfields of linguistics and related disciplines, each of these focussing on particular aspects of this phenomenon. Through the use of Coseriu's theory, it is possible to refine our understanding of 'expressions with the same or similar meaning' and to provide a coherent description of the causes and the effects arising from the choice between them in texts that may otherwise remain non-transparent and inexplicable. This paper presents a method for analyzing the actual relationships between such expressions in a corpus, a way of exploring their functions in texts, and some possible benefits for understanding the notion of synonymy as well as for understanding implicitly conveyed information in texts.
\end{abstract}

Fondé sur la thèse de doctorat de l'auteur, cet article présente la méthodologie suivie et les résultats obtenus d'une application de la linguistique Coserienne à la recherche de la synonymie. Cette thèse, publiée sous le titre $O$ sinonimiji [Sur la synonymie] ${ }^{1}$, a eu pour but d'élaborer une méthode d'analyse des relations entre des expressions synonymes au sein d'un corpus de textes, et de décrire comment le choix des synonymes influence le sens du texte.

La synonymie est communément reconnue comme étant un rapport d'identité ou de proximité sémantique entre des expressions linguistiques. Pourtant, il n'existe aucune définition satisfaisante de ce phénomène pluridimensionnel, bien qu'en plus d'avoir été l'objet d'une étude intensive de la sémantique, la synonymie a suscité beaucoup d'intérêt en rhétorique, en stylistique, en linguistique textuelle, en sociolinguistique, en pragmatique, en analyse du discours et en philosophie. Les définitions de la synonymie proposées par les représentants de diverses théories sémantiques ont provoqué des controverses au sujet du concept même de synonymie $^{2}$. L'une de ces controverses réside dans le fait de savoir si la synonymie est un fait du système langagier ou seulement un phénomène observable dans des textes ; une autre controverse concerne l'identification des critères appropriés pour déterminer la nature de la synonymie ou encore si la similarité sémantique peut être une question de degré. C'est pourquoi le choix des fondements théoriques est d'une importance capitale pour une description de la synonymie conforme à la réalité langagière. Dans cette optique, la pensée linguistique de Coseriu nous offre un bon point de départ pour trois raisons.

1. D'abord, la théorie linguistique de Coseriu (1973: 9 sqq., 1985: 42-48 ou 1992: 262-264) réunit les trois types de relations qui sont en jeu dans la synonymie, c'est-à-dire :

\footnotetext{
1 cf. Zorman 2000a.

2 Pour différentes tentatives de définition de la notion de synonymie, cf. Carnap (1955: 34), Quine (1960: 65), Filipec (1961: 203), Katz et Fodor (1963: 184 sq.), Zvegincev (1963: 132), Ducháček (1964: 37), Gabka (1967: 728), Ullmann (1967: 102), Bierwisch (1970: 170), Harris (1970: 786), Mel'čuk et Žolkovskij (1970: 45), Gauger (1972: 436), Harris (1973: 1-20), Nida (1975: 233), Apresjan (1980), Lyons (1963: 58, 1975: 460, $1980: 302$ et 1981: 94), Cruse (1986), Rey-Debove (1997), Murphy (2003: 133 sq.) etc. Pour une discussion détaillée des problèmes définitoires et des controverses, voir Zorman (2000a: 20-37).
} 
a) La relation entre un signe linguistique et un objet $^{3}$;

b) La relation entre un signe linguistique et d'autres signes linguistiques ;

c) La relation entre un signe linguistique et le contexte linguistique et situationnel.

Ces relations sont en effet abordées dans toutes les études sur la synonymie mais apparaissent sous des désignations différentes comme la référence, la dénotation, l'intension, l'extension, certains concepts du sens, etc. ${ }^{4}$ La première d'entre elles - la relation entre un signe linguistique et un objet - était déjà connue par Aristote 5 . Aujourd'hui, les théories sémantiques postulant que le sens constitue une représentation mentale des phénomènes du monde extérieur, notamment la sémantique référentielle ou vériconditionnelle, se sont basées sur cette relation ${ }^{6}$. Coseriu aborde la relation entre un signe et un objet au niveau de la dénomination. Un avantage du concept de dénomination chez Coseriu par rapport à des théories sémantiques indépendantes du contexte comme par exemple l'analyse traditionnelle de la signification, l'analyse componentielle ou la sémantique vériconditionnelle ${ }^{7}$, est que celles-ci abordent cette relation au niveau de la langue, tandis que le concept de dénomination chez Coseriu se situe au niveau du discours ${ }^{8}$.

L'existence d'une relation entre un signe linguistique et d'autres signes linguistiques est la condition nécessaire et logique de toute relation de synonymie. Chez Coseriu ${ }^{9}$, ce concept est inclus dans la notion de signification qui est déterminée par la relation structurelle entre les signifiés des divers signes. Ce domaine a été un objet courant d'étude de la sémantique paradigmatique, mais il existe cependant des divergences importantes entre Coseriu et d'autres représentants ${ }^{10}$ de la sémantique paradigmatique, tant sur le plan de la terminologie que sur la nature et l'étendue des possibles configurations de significations envisagées par les uns et les autres $^{11}$.

Enfin, la relation entre un signe linguistique et le contexte ou le texte joue un rôle central dans toute notion dépendante du contexte de la synonymie et est un objet d'étude dans la pragmatique (voir Hundsnurscher 1992: 292) et la stylistique (Filipec 1961: 45-170), ainsi que dans les approches défendant l'idée que « la signification, c'est l'usage ${ }^{12}$. La théorie de Coseriu a l'avantage de pouvoir démontrer comment le sens d'une expression qui apparaît au niveau du texte dépend du contenu situé à des niveaux englobants ${ }^{13}$.

\footnotetext{
${ }^{3}$ Le terme « objet » s'entend ici au sens de toutes les entités et faits extralinguistiques, qui font partie du monde perceptible ou concevable.

4 Voir Lyons (1980: 189 et 219-228) pour la dénotation, Lyons (1981: 157) pour l'intension et l'extension, et ibid. p. 58 sqq. pour le sens. Pour une discussion des différentes compréhensions de ces notions et du rapport entre elles, voir Zorman (2000a: 33, 51 et 67).

5 Selon Coseriu (2003: 65-108), Aristote distingue la signification propre du mot (páthema) de l'objet désigné (pragma, la référence). Dans sa conception, ónoma unit le son et le contenu et désigne une chose. Cette distinction correspond à la distinction entre la forme linguistique, la signification et la dénomination chez Coseriu. A ce sujet, voir aussi Haßler (à paraître: 2 sq.) et De Cuypere et Willems (2008: 307-324).

${ }^{6}$ Sur leur assimilation à la réflexion aristotélicienne, voir Rastier (1990).

${ }^{7}$ Pour plus d'informations sur ce sujet, voir Rastier, op. cit.

8 Pour une discussion détaillée de ces questions, cf. Coseriu (1970: 44 sq.).

9 Voir par exemple Coseriu (1973: 9 sqq., 1985: 42-48 ou 1992: 262-264).

10 Cf. Nida (1975: 15-22, 68-110) et Lyons (1963: 58, 1980: 302 et 1981: 94).

11 Pour diverses positions théoriques sur les relations sémantiques paradigmatiques et un résumé de la recherche sur ce sujet, voir Murphy (2003). Cf. aussi Coseriu (1970: 8-47).

12 Ces approches sont fondées sur la théorie de Wittgenstein selon laquelle La signification d'un énoncé, c'est son usage syntaxique qui a eu des échos en analyse distributionnelle des significations ; cf. Apresjan (1966) et Eckert (1968).

13 Coseriu (1985) expose sa conception du sens dans son introduction à la linguistique du texte.
} 
2. Le deuxième avantage de la pensée linguistique de Coseriu pour éclairer le problème de la synonymie réside dans sa conception élargie de la notion de langue, qui comprend à la fois un système fonctionnel et une réalisation habituelle et traditionnelle, c'est-à-dire la norme ${ }^{14}$. En effet, si nous concevons la norme comme un phénomène relevant de la parole, comme le faisaient l'Ecole de Prague et celle de Copenhague (Coseriu 1975: 58-62), la synonymie se verrait reléguée au rang d'objet d'étude dépendant de la stylistique du mot ou du texte, ce qui conduirait à la conclusion qu'il n'existe pas de synonymes, parce que la norme favorise toujours l'un d'entre eux.

3. Le troisième avantage de la théorie de Coseriu pour l'élucidation de la synonymie est sa distinction entre la structure et l'architecture de la langue et, par conséquent, entre les oppositions et les diversités lexicales ${ }^{15}$. Si, par exemple, une expression d'un dialecte quelconque est perçue comme un élément « normal » et neutre du système lexical du dialecte et non par opposition au lexique d'une langue historique, il n'est plus possible de faire la distinction dans ce cas entre, d'une part, les synonymes neutres et, de l'autre, les synonymes marqués stylistiquement, ou également entre des synonymes descriptifs ${ }^{16}$ d'un côté et affectifs de l'autre côté ${ }^{17}$. Puisqu'il est important dans la théorie coserienne de ne pas confondre les systèmes, on ne doit pas comparer différents types de synonyme entre eux. Ainsi, « le rapport d'identité ou proximité sémantique » devient plus transparent.

En nous inspirant des propositions de Coseriu concernant l'étude des divers niveaux de contenu d'un mot, nous proposons la stratégie d'analyse suivante pour étudier l'usage des synonymes dans un corpus :

i. Identifier la langue fonctionnelle ${ }^{18}$ du texte où se trouve le mot en question ;

ii. Déterminer une dénomination pour chaque occurrence du mot. Cette tâche consiste à identifier la variante lexicale en utilisant un procédé de paraphrase (Coseriu 1973: 11)

\footnotetext{
14 Voir par exemple Coseriu (1975: 62).

15 Dans la conception coserienne, il faut faire une distinction entre la structure et l'architecture de la langue aussi bien qu'entre la langue historique et la langue fonctionelle. Une langue historique comme le français ou l'allemand n'est jamais un seul «système linguistique » et la «technique synchrone de discours » dans une telle langue n'est pas d'une nature homogène. Cette technique synchrone de discours met à jour des différences internes dans l'espace géographique (différences diatopiques), des différences de nature socioculturelle (différences diastratiques) et des différences entre les types de modalité expressive (différences diaphasiques). Coseriu appelle l'ensemble des rapports que comporte la multiplicité des «techniques du discours » coexistantes d'une langue historique l'architecture de la langue, tandis que la structure de la langue concerne exclusivement les rapports entre les termes d'une «technique du discours » déterminée (langue fonctionnelle). «Entre les termes «différents» du point de vue de la structure de la langue, il y a opposition; entre les termes «différents» du point de vue de l'architecture de la langue, il y a diversité. Ainsi, le fait que ami et camarade sont des termes «différents » (c'est-à-dire qu'ils ne signifient pas «la même chose ») dans le français moyen est un fait de structure, une opposition. Par contre, le rapport entre les termes ami, camarade du français moyen et le terme copain du français populaire (et familier) est un fait d'architecture de la langue, une diversité. » (Coseriu 2001: 241-242, cité d'après Missire (2006: 40).

16 Nommés aussi synonymes cognitifs.

17 Pour une tentative d'élaboration d'une telle typologie de synonymes, voir Ducháček (1964). D'après Ducháček, le mot animal, par exemple, est complètement dépourvu de toute notion de sensibilité (il est donc neutre), tandis que bête peut contenir l'expresion d'une certaine sympathie et peut être classé comme un synonyme affectif d'animal. Les synonymes péjoratifs d'avare sont avaricieux, crasseux, sordide, rat et chien. Malheur et malheureux n'ont pas l'air aussi noble qu'infortune et infortuneux, c'est pourqui il les appelle des «synonymes fonctionels ». Au contraire, la paire de synonymes bien que - quoique lui semble parfaite et interchangeable en toutes circonstances. Pour d'autres essais typologiques, cf. Ullmann (1967: 102) et Filipec (1961: 201-203).

18 Une langue fonctionnelle est « une technique du discours homogène conjoignant unité syntopique, synstratique et synphasique ». (Cité d'après Missire (2006: 40.) Voir aussi Coseriu (1975: 58-62).
} 
et à identifier les déterminants nominaux ainsi que les entours ${ }^{19}$ (Coseriu 1975: 253290) ;

iii. Éliminer les exemples du discours répété, d'une part, et identifier, d'autre part, la relation entre les mots en question dans les systèmes des langues fonctionnelles du corpus en utilisant un procédé de commutation ;

iv. Décrire les propriétés catégorielles du mot et sa motivation (Hjelmslev 1980: 62 sqq.);

v. Déterminer le sens des mots qui sont l'objet de l'étude au moyen d'une analyse de leur distribution dans le corpus (Coseriu 1970: 56 et Heringer s. a.) ;

vi. Déterminer le type de texte où se trouvent les mots et juger si le synonyme sélectionné correspond à la norme du texte (Mistrík 1975) ;

vii. Rechercher les évocations ${ }^{20}$ du synonyme sélectionné ainsi que sa contribution au sens de l'ensemble du texte.

En pratique, nous nous sommes trouvés contraints, vu le manque d'analyses linguistiques approfondies de la langue slovène (objet de notre recherche), de simplifier le procédé et d'avoir recours à des jugements intuitifs lors de certaines étapes comme, par exemple, l'identification de la langue fonctionnelle d'un texte ou lorsque nous devions déterminer si un mot fait partie du système lexical d'une langue fonctionnelle ou non. Lors d'autres étapes, il a été nécessaire de compléter la théorie de Coseriu à l'aide d'idées puisées dans la sémantique distributionnelle et la linguistique textuelle afin de la rendre applicable. Tel a été le cas, par exemple, pour passer de l'étape de la description de l'usage des synonymes dans des textes à celle de la détermination de leurs relations au sein du système de la langue. D'après Coseriu (1970: 56), il est possible d'identifier la place du lexème dans le système lexical à partir de sa distribution. Afin d'élucider la distribution des lexèmes, nous avons recherché tous les environnements contextuels d'un mot dans un corpus ${ }^{21}$ de plus de six millions de mots à l'aide de la méthode de sémantique distributionnelle développée par Hans Jürgen Heringer. En collaboration avec M. Ohlenroth de l'Université d'Augsburg, Heringer a conçu un programme CIA (Computer Image Analyse) ${ }^{22}$ pour l'analyse assistée par ordinateur des mots dans un corpus de textes. Dans la première étape, le concordancier a recherché une chaîne de recherche ${ }^{23}$ dans un contexte donné et a établi une liste de toutes les occurrences du mot avec une partie du contexte antérieur et postérieur, dont l'étendue était précisée dans les paramètres de la recherche ; les exemples (1)-(3) représentent quelques extraits d'une de ces listes :

(1) občinskemu organu omogoči presojo, ali je reflektant upravičen do želenega državljanstva ali ne. Torej, ali bo to odvisno od trenutnih simpatij (kuvert) ali nesimpatij do človeka, ki pol stoletja sploh ni vedel za to premoženje (na primer nasledniki razlaščencev). Na primer trgovec

'il permet à l'organe communal de juger si l'intéressé a le droit d'acquérir la nationalité ou non. Ce fait dépendrait-il donc de la sympathie (enveloppe) ou de l'antipathie actuelle envers la

\footnotetext{
19 L'entour dans la conception coserienne est l'« ensemble des phénomènes sémiotiques associés à une suite linguistique ; plus généralement, contexte non linguistique, dit parfois pragmatique ». (Cité d'après Rastier 1989: 278.)

${ }^{20}$ Le concept coserien d'évocation comporte l'ensemble des fonctions associatives du signe linguistique dans le texte ; voir p. ex. Coseriu (1985: 102).

${ }^{21}$ Sur la structure du corpus, voir note 30 infra.

22 Une version plus récente de ce programme (développée à Ludwigs-Universität-Freiburg par R. Widdig et K. Müller) a été employée dans le livre Das höchste der Gefühle (Heringer 1999) ; cf. aussi Heringer (2010). Pour une présentation détaillée de ce programme, le lecteur peut se référer à http://www.philhist.uniaugsburg.de/Faecher/GERMANIS/daf/neu/heringer/ins01.php, consulté le 14 novembre 2012.
} 
personne qui pendant cinquante ans n'a eu aucune connaissance de ces biens (par exemple les descendants des expropriés). Un commerçant, par exemple'

(2) Največ sporov nastane zaradi nespoštovanja kolektivnih pogodb. Vedno več je delavcev z najnižjimi plačami, ki že od septembra niso prejeli kuverte. Če bodo legitimne poti za uveljavljanje delavskih pravic še naprej blokirane, bo delavske strpnosti konec, kar pa ne bo dobro

'La majorité des conflits naissent de l'irrespect des conventions collectives. Il y a de plus en plus d'ouvriers ayant les salaires les plus bas qui n'ont pas eu d'enveloppe depuis septembre. Si les moyens légitimes pour faire respecter les droits d'ouvrier restent toujours bloqués, la tolérance ouvrière ne perdurera pas, ce qui risque de ne pas bien finir'

(3) in se ni smejal. "Tudi jaz sem povedal očetu. Vesel ravno ni bil." V hipu je potegnil iz žepa zmečkano kuverto in mi jo ponudil z besedami: "Tole mi je dal oče. Na, vzemi!" Segla sem po modri ovojnici in jo

'et il ne riait pas. «Moi aussi, je l'ai dit à mon père. Et il n'a pas été heureux. » A l'instant, il tira de sa poche une enveloppe froissée et me l'offrit en disant : «Mon père m'a donné ceci. Tiens! » Je tendis la main pour prendre l'enveloppe bleue et je l'ai'

Le programme a, par la suite, éliminé la structure grammaticale des contextes en supprimant les mots fonctionnels et en remplaçant toutes les désinences flexionnelles par la désinence de base $^{24}$. Cette opération a transformé les exemples (1)-(3) en (1')-(3') :

(1') občinski 'communal'

organ 'organe'

omogočiti 'permettre'

presoja 'jugement'

reflektant 'intéressé'

upravičen 'ayant le droit'

želen 'souhaité'

državljanstvo 'nationalité'

odvisen 'dépendant'

trenuten 'momentané, actuel'

simpatija 'sympathie'

kuverta 'enveloppe'

(2') veliko 'beaucoup'

spor 'conflit'

nastati 'commencer', 'naître' nesimpatija 'antipathie'

človek 'homme', 'personne'

pol 'demi'

stoletje 'siècle'

vedeti 'avoir connaissance'

premoženje 'avoir', 'bien', 'fortune'

primer 'exemple'

naslednik 'descendant'

razlaščenec 'exproprié'

primer 'exemple'

trgovec 'commerçant'

prejeti 'avoir', 'recevoir', 'obtenir'

kuverta 'enveloppe'

legitimen 'légitime'

\footnotetext{
23 Pour améliorer les résultats des recherches, nous avons évité d'utiliser des mots complets en tant que chaîne de recherche. Nous avons indiqué par exemple jnic pour obtenir ovojnica.

24 C'est-à-dire que, par exemple, les variations flexionnelles ovojnice, ovojnici, ovojnico, ovojnic, ovojnicam, ovojnicah, ovojnicama, ovojnicami ont été ramenées à leur forme canonique ovojnica.
} 
nespoštovanje 'irrespect'

kolektiven 'collectif'

pogodba 'convention'

vedno 'toujours'

veliko 'beaucoup'

delavec 'ouvrier' (subst.)

nizek 'bas'

plača 'salaire'

september 'septembre'

(3') smejati 'rire'

povedati 'dire'

oče 'père'

potegniti 'tirer'

žep 'poche'

zmečkan 'froissé'

kuverta 'enveloppe'

ponuditi 'offrir'

beseda 'parole', 'mot' pot 'voie'

uveljavljanje 'revendication'

delavski 'ouvrier' (adj.)

pravica 'droit' (subst. masc.)

naprej 'en avant'

blokiran 'bloqué'

delavski 'ouvrier' (adj.)

strpnost 'tolérance'

konec 'fin'

dobro 'bien'

vesel 'heureux', 'joyeux', 'gai'

ravno 'juste', 'justement'

hip 'moment', 'instant'

dati 'donner'

oče 'père'

vzeti 'prendre'

seči 'tendre la main'

moder 'bleu'

ovojnica 'enveloppe'

À l'étape finale, le programme fournit une «synthèse » des contextes pour lesquels il a calculé la moyenne de fréquence et de distance relative des mots qui apparaissaient dans des contextes contenant le mot recherché. Le résultat se présente sous la forme d'un tableau ou d'un graphique ${ }^{25}$. Il a fallu adapter ce programme pour traiter la langue slovène. Les travaux initiaux ont donc consisté à établir une liste de mots fonctionnels slovènes et à élaborer un dictionnaire de lemmatisation. La synthèse a indiqué les mots apparaissant le plus souvent près du mot recherché dans les textes du corpus. Heringer supposait que si le corpus était assez grand, il pourrait représenter la structure sémantique d'un mot dans la langue sans qu'y figurent des mots particuliers à un texte. Cela n'a pas du tout été le cas lors de notre analyse. Notre corpus était trop petit et la plupart des mots inclus dans les synthèses étaient particuliers aux textes ${ }^{26}$. Pourtant, bien qu'ils soient particuliers aux textes, ils se sont avérés utiles pour l'analyse des structures paradigmatiques des mots étudiés. La synthèse du mot ovojnica 'enveloppe' incluait, par exemple, les expressions de couleur bel 'blanc' et zlat 'doré' ; il était évident dans le cas de 'doré' que cet attribut ne pouvait pas être caractéristique du mot enveloppe dans le système langagier. Néanmoins, notre raisonnement a été le suivant : si

\footnotetext{
${ }^{25}$ Voir p. 10 et 11 infra; cf. aussi Zorman (1998b), Muroi (1998) et Heringer (1999).

${ }^{26}$ Sur ce problème, voir la discussion des mots bel, zlat, cilj, papir, etc. infra et les propositions supplémentaires dans Zorman (2005a: 331-333).
} 
quelque chose est pourvu d'une couleur, il doit appartenir à la classe des objets concrets ${ }^{27}$. L'apparition du mot cilj 'visée' dans les synthèses des mots borba et boj 'lutte', semblait justifier le fait de ranger les mots relatifs à la lutte dans la classe des noms désignant les activités intentionnelles. Les mots qui désignent des matériaux tels que papir 'papier' tkivo 'tissu', tkanina 'étoffe', plastika 'plastique' ont dévoilé des oppositions dans les champs sémantiques de ovitek 'pochette', ovoj 'bandage' et plastična embalaža 'emballage plastique', par exemple. De cette manière, il a été possible de déterminer la classe et le champ sémantique dans lesquels on pouvait ranger les synonymes étudiés.

Pendant l'étape d'identification de la norme d'un texte concernant le choix d'un synonyme, la théorie de Coseriu a dû être complétée par une typologie des textes qui tient compte du choix des mots. Nous nous sommes servis de l'analyse de textes professionnels et journalistisques de Mistrík (1975), qui décrit les aspects des textes qui sont des indicateurs potentiels du statut normatif ou non d'un synonyme donné. Mistrík a noté le degré de polysémie potentielle des mots et a constaté que, dans certains types de textes, comme, par exemple, dans les articles scientifiques ou dans les règlements, les mots devraient être monosémiques et leur signification indépendante du contexte. D'autres textes (reportages journalistiques, légendes, certains textes d'humour, etc.) permettent un usage modéré des métaphores mais désambiguïsent des mots polysémiques, tandis que dans d'autres textes (surtout dans les textes de genre litteraire), il n'est possible de comprendre certains mots qu'à partir d'une compréhension de l'ensemble du texte. Par ailleurs, Mistrík a examiné le degré de subjectivité du style ${ }^{28}$ et les intentions de l'auteur (fournir des informations, des explications, ou atteindre un but artistique). Nous nous sommes servis des catégorisations de Mistrík de divers types de textes $^{29}$ pour étayer nos évaluations intuitives concernant la fonction du choix entre les synonymes dans un texte quelconque.

Après avoir résolu les problèmes d'ordre méthodologique, nous avons utilisé le procédé présenté supra pour analyser chaque occurrence de deux paires de mots slovènes dans le corpus mentionné ${ }^{30}$. Ces deux paires de mots étaient borba-boj et kuverta-ovojnica. Dans le dictionnaire normatif du slovène standard de l'Académie slovène (SSKJ I: 164 et 173 sq.) borba et boj sont donnés comme synonymes complets avec le sens de 'combat' ou 'lutte'. Cependent, kuverta et ovojnica sont y tenus pour synonymes partiels ${ }^{31}$ dans la mesure où le premier a le sens unique d'enveloppe de papier à lettres' (SSKJ II: 535), tandis que le deuxième désigne aussi d'autres sortes d'emballage (SSKJ III: 490). Nous présentons cidessous les synthèses des contextes des mots kuverta et ovojnica ((4) et (5)), un exemple illustrant l'application de la méthode d'analyse proposée en points i-vii, p. 3-4 supra (exemple

\footnotetext{
${ }^{27}$ D'après Coseriu (1970: 8-9 et 27-32), les expressions comme idées noires ou peurs bleues ne peuvent pas faire partie d'une description structurale de la signification. On doit tenir compte de leur contenu au niveau de la dénomination et pas dans une analyse des structures paradigmatiques.

${ }^{28}$ Selon Mistrík, le style est subjectif si l'auteur est présent dans son texte et si les destinataires peuvent identifier son ton (par exemple l'humour, l'ironie, l'indignation).

${ }^{29}$ Dans sa classification, les instructions administratives, par exemple, représentent par rapport au choix lexical un genre informatif objectif dont l'auteur ne peut pas être identifié. Le style des instructions administratives doit être précis et neutre et la seule intention percevable de l'auteur doit être celle d'informer. Ce genre ne permet pas de formulations inhabituelles, pas de mots expressifs et pas de métaphores, tandis qu'un usage modéré des mots polysémiques est acceptable. La structure grammaticale se caractérise par l'usage fréquent de formes modales et d'auxiliaires de mode.

${ }^{30}$ Un corpus écrit du slovène contemporain (environ $40 \mathrm{Mb}$ ) a été créé par l'auteur. Il a été constitué de textes scientifiques, administratifs, journalistiques et littéraires, de textes spécialisés et de textes de la langue courante publiés entre 1990 et 1995.

${ }^{31} \mathrm{La}$ 《 synonymie complète » s'entend ici comme la propriété qu'ont les mots avec des sens complètement identiques, tandis que la « synonymie partielle » est la propriété que présentent les mots qui partagent un ou plusieurs sens mais divergent également pour certains autres. Cf. aussi Lyons (1981: 50-51) et Ducháček (1964: 9).
} 
(6)) ainsi qu'un bref résumé de nos observations concernant les relations et la fonction du choix entre les synonymes recherchés :

(4) La synthèse des contextes du mot kuverta

$\begin{array}{llll}\text { bančen 'bancaire' } & 6 & \text { kuverta 'enveloppe' } & 2 \\ \text { četrtek 'jeudi' } & 6 & \text { lestvica 'échelle' } & 5 \\ \text { dati 'donner' } & 8 & \text { list 'feuille' } & 3 \\ \text { dodaten 'supplémentaire' } & 8 & \text { minister 'ministre' } & 6 \\ \text { italijanski 'italien' } & 8 & \text { moder 'bleu' } & 5 \\ \text { nakup 'achat' } & 6 & \text { praviti 'dire', 'raconter' } \\ \text { nazaj 'en arrière' } & 5 & \text { prejeti 'recevoir' } & 6 \\ \text { način 'façon, manière', 'mode' } & 8 & \text { prinesti 'apporter' } & 7 \\ \text { občina 'municipalité', 'comune' } & 6 & \text { prispeti 'arriver', 'venir' } & 3 \\ \text { oddati 'remettre', 'envoyer' } & 6 & \text { ugotoviti 'constater', 'établir' } & 2 \\ \text { odpreti } \text { 'ouvrir' } & 3 & \text { večinski 'majoritaire' } & 6 \\ \text { otrok 'enfant' } & 4 & \text { vzeti 'prendre' } & 8 \\ \text { papir 'papier' } & 3 & \text { začeti 'commencer' } & 6 \\ \text { plača 'salaire' } & 7 & \text { zanimati 'intéresser' } & 6 \\ \text { ponudba 'offre' (subst.) } & 2 & \text { zapečatiti 'cacheter', 'sceller' } & 3 \\ \text { ponuditi 'offrir', 'proposer' } & 5 & \text { zapreti 'fermer' } & 4 \\ \text { poseben 'particulier', 'spécial' } & 7 & \text { zatrditi 'assurer', 'affirmer' } & 1 \\ \text { potegniti 'tirer' } & 6 & \text { zložiti 'composer', 'mettre ensemble' } & 5 \\ \text { povedati 'dire' } & 4 & \text { zmečkati 'écraser, froisser' } & 6 \\ & & & 5\end{array}$

(5) La synthèse des contextes du mot ovojnica

$\begin{array}{llll}\text { bel 'blanc' } & 14 & \text { naprej 'en avant' } & 13 \\ \text { boleč 'douloureux' } & 11 & \text { noga 'jambe' } & 14 \\ \text { bolečina 'douleur' } & 12 & \text { odprtina 'ouverture', 'trou', 'fente' } & 8 \\ \text { delo 'travail' } & 10 & \text { ovojnica 'enveloppe' } & 4 \\ \text { disketa 'disquette' } & 15 & \text { ozek 'étroit' } & 9 \\ \text { glava 'tête' } & 14 & \text { papir 'papier' } & 15 \\ \text { golen 'bas de la jambe' } & 9 & \text { pisemski 'épistolaire', 'à lettres' } & 10 \\ \text { golenica 'tibia' } & 11 & \text { poškodba 'lésion' } & 4 \\ \text { imeti 'avoir' } & 12 & \text { predstavitev 'présentation' } & 11 \\ \text { iti 'aller' } & 15 & \text { sindrom 'syndrome' } & 5\end{array}$




$\begin{array}{llll}\text { izvirati 'découler (de qc.)' } & 10 & \text { sklep 'articulation', 'résolution' } & 12 \\ \text { kita 'tendon' } & 3 & \text { sklepen 'articulaire' } & 1 \\ \text { kontakten 'de contact' } & 13 & \text { spraviti 'garder', 'conserver' } & 17 \\ \text { mečnica 'péroné' } & 17 & \text { telo 'corps' } & 13 \\ \text { mišica 'muscle' } & 7 & \text { tesen 'serré' } & 4 \\ \text { mišičen 'musculaire' } & 3 & \text { tkivo 'tissu' } & 11 \\ \text { moder 'bleu' } & 13 & \text { varovati 'préserver', 'protéger' } & 11 \\ \text { vez 'ligament' } & 5 & \text { vsebovati 'contenir', 'renfermer' } & 16 \\ \text { vnetje 'inflammation' } & 3 & \text { zabrazgotiniti 'cicatriser' } & 13 \\ \text { vpliv 'influence', 'impact' } & 12 & \text { zlat 'doré' } & 10\end{array}$

(6) L'analyse du mot ovojnica dans un écrit officiel adressé à un collègue

\section{Zdravo!}

Vesel potrjujem svojo udeležbo na raziskovalnem taboru na Kozjaku. Na vse kriplje nabiram potrebno kondicijo (telesno, umsko ...), potrebno za delo v Tvoji skupini. Vse dobro in lep pozdrav!

$$
\text { R.P. }
$$

P. S.: Ker je "priloženo pismo" sestavljala le modra ovojnica, sem pismo napisal sam in ga poslal v originalni ovojnici.

'Salut!

J'ai le plaisir de confirmer ma participation au camp de recherche sur le Kozjak. Je me mets en quatre pour être en meilleure forme (physique, mentale), nécessaire pour le travail dans ton groupe. Bon courage et amitiés!

$$
\text { R.P. }
$$

P.S. : Puisque « la lettre jointe » ne consistait que de l'enveloppe bleue, j'ai écrit la lettre moimême et l'ai envoyée dans l'enveloppe originale.'

i. Langue fonctionnelle : langue courante ;

ii. Dénomination : ovojnica désigne ici une 'enveloppe de papier à lettres' (paraphrase : pisemski ovitek 'enveloppe de papier à lettres' ; déterminant nominal restreignant les références possibles de ce mot dans le contexte : modra ovojnica 'enveloppe bleue') ;

iii. Discours répété : ce texte ne comporte pas d'expressions phraséologiques appartenant à la catégorie du discours répété32 ; commutation (ker je »priloženo pismo" sestavljala le modra kuverta, sem pismo napisal sam in ga poslal v originalni kuverti) démontre qu'à en juger uniquement par ce texte ovojnica et kuverta pourraient être censés le même invariant dans langue courante ; entour : correspondance ; contexte verbal : pismo 'lettre' ;

iv. Propriétés catégorielles : ovojnica est un substantif féminin dérivé du verbe oviti 'entourer pour protéger, en couvrant entièrement à l'aide de quelque chose', c'est un mot slovène d'origine indo-européenne, alors un mot indigène ;

\footnotetext{
32 Voir par exemple Coseriu (1970: 27-32).
} 
v. Le sens de ovojnica dans le corpus : 'partie externe, protectrice de quelque chose en papier, tissu, etc.' ;

vi. Type de texte : lettre semi-officielle; norme du texte : bien que la norme de la langue courante favorise le mot kuverta, l'auteur d'une lettre semi-officielle est libre de choisir une expression qui la contrarie ${ }^{33}$;

vii. Contribution au sens du texte : l'expression ovojnica appartenant aux registres soutenu et littéraire est indicatrice de la politesse distante de l'auteur.

En appliquant cette méthode d'analyse à toutes les occurrences des mots borba, boj, kuvera et ovojnica dans notre corpus, nous avons pu donner une description détaillée des propriétés sémantiques, des rapports et de l'usage de ces deux paires de mots. Les relations entre les «synonymes complets » ou bien «partiels » se sont avérées extrêmement variables et complexes. Vu l'impossibilité de rendre compte des résultats de manière détaillée dans le présent article, nous nous limitons à la présentation d'un bref résumé de nos observations ${ }^{34}$.

Au niveau de la dénomination, l'analyse a démontré que même des mots qui sont censés être monosémiques comme kuverta ont en fait des dénominations multiples. Ils peuvent être utilisés d'une façon métaphorique ou métonymique ${ }^{35}$; nous ne voyons, en effet, aucune distinction en pratique entre les mots monosémiques et polysémiques ${ }^{36}$. La structure de la dénomination est bien sûr variable dans des langues fonctionnelles différentes et certaines langues fonctionnelles ont même tendance à utiliser des mots différents pour désigner la même chose. La commutation (cf. Zorman 2000a: 84 sq., 100 sq., 2005a) a démontré que, dans certains systèmes fonctionnels, deux synonymes peuvent être perçus comme identiques dans leur signification, tandis que dans d'autres systèmes il y a une opposition sémantique entre ces mêmes mots ${ }^{37}$. Il arrive aussi qu'un seul des mots soit présent dans le système lexical. Si deux mots ou plus représentent le même invariant, le choix du mot dépend de la norme du texte. Il est possible que la norme favorise l'un ou l'autre, mais chacun a la possibilité d'être utilisé fonctionnellement ${ }^{38}$; nous n'avons donc pas pu confirmer l'hypothèse qu'il existe des synonymes neutres, d'une part, et des synonymes marqués stylistiquement d'autre part. Par conséquent, il semblerait inapproprié de tenter une catégorisation de la synonymie. Dans certains types de textes (par exemple dans des notices, modes d'emploi,

\footnotetext{
33 Cf. Brinker (1985: 128 sq.).

34 Pour une présentation plus détaillée des resultats obtenus, le lecteur est prié de se référer à Zorman (2000a: 79-109, 2000b et 2005a).

35 En plus de désigner une 'enveloppe de papier à lettres' kuverta s'utilise dans notre corpus aussi dans le sens figuré pour la désignation d'une 'lettre', d'un 'pot-de-vin', d'un 'salaire', d'une 'offre' et d'un 'décret' ; voir Zorman (2000a: 79-81). Selon le dictionnaire de l'Académie slovène (SSKJ II: 535), c'est un mot monosémique qui peut être utilisé aussi dans deux constructions idiomatiques (vsakega prvega ji da kuverto 'au début de chaque mois, il lui donne son salaire complet' et prejemal je težke kuverte 'il recevait de grands pots-de-vin').

36 A ce propos, la comparaison entre le mode d'emploi dans notre corpus et la description lexicographique des mots kuverta et borba peut être illustratif. Comme kuverta (voir note 35 supra), borba s'utilise aussi pour désigner plusieurs choses. D'après nos données, cela peut être une 'opération militaire' et dans le sens figuré encore une 'lutte d'idées', une 'action de prendre parti pour une cause, une doctrine', etc. ; voir Zorman (2000a: 92-95). Selon SSKJ (I: 164), ce sont trois sens propres de ce mot et à la différence de kuverta, il passe pour un mot polysémique. Dans de tels cas, les critères de la démarcation entre les mots monosémiques et les mots polysémiques ne sont pas évidents. (Pour la discussion de la délimitation de la polysémie par rapport à des variantes contextuelles de la désignation chez Coseriu, cf. Dupuy-Engelhardt (1990: 15 sqq.))

37 Ainsi, dans la langue des textes journalistiques, les mots kuverta et ovojnica peuvent toujours être commutés, ils sont donc des variantes au niveau de la norme, mais ils présentent une seule invariante au niveau du système de cette langue. Dans la langue litteraire, ovojnica a une signification plus ample que kuverta et il y a des attestations dans lesquelles ils ne sont pas commutables.

38 Voir Zorman (2000a: 91 sq., 108 sq., 2000b: 34-41). Par exemple, l'emploi du mot borba peut évoquer une déficience de compétence expressive, renvoyer au discours politique ou susciter une association d'idée avec une course de taureaux (bikoborba). Par contre, boj a la capacité d'évoquer l'ambiance marxiste.
} 
comptes rendus ou articles scientifiques), la norme est prédéterminée mais dans d'autres (reportage journalistique, pamphlet, comédie, etc.) ce n'est pas le cas (Zorman 2000a: 129, 2000b: 32 sq.). Si la norme est préétablie, l'auteur peut la suivre ou rompre avec elle, que ce soit à dessein ou involontairement. Dans ce cas, une analyse des textes révèle souvent les intentions de l'auteur même si celles-ci ne sont pas explicites. L'auteur peut rompre avec la norme en employant des expressions hypercorrectes ou non-standard ; de tels usages sont révélateurs surtout de l'identité sociale ou de l'état psychologique de l'auteur et reflètent souvent des relations de pouvoir entre interlocuteurs (Zorman 2000b: 34-36). Les déviations intentionnelles par rapport à la norme appartiennent au discours manipulatoire ${ }^{39}$. Une des techniques les plus subtiles de manipulation est de choisir un mot neutre qui puisse évoquer notre connaissance de la réalité extralinguistique. Contrairement aux textes où il est attendu que l'auteur obéisse à la norme, il existe des textes ${ }^{40}$ où l'auteur doit opérer un choix fonctionnel et ainsi moduler le sens du texte. Dans de tels textes ${ }^{41}$, il n'existe pas de règles qui déterminent la sélection d'un mot ; l'auteur peut profiter de cette liberté et exploiter le potentiel fonctionnel d'un mot (Zorman 1998a, 2000a: 129-130). Ce faisant, il peut se servir librement de la variation lexicale, des métaphores, des métonymies et du langage figuré existant. Il peut aussi se reposer sur les relations entre des mots et des objets pour construire le sens du texte. Il peut se servir de tels choix pour établir ou influencer des relations entre les personnes, pour exprimer implicitement des sentiments ou des points de vue, pour renforcer l'argumentation ou pour gérer la structure du discours. Afin de prévenir la révélation d'une évocation, certaines conditions nécessaires doivent prévaloir (cf. Zorman 2000b: 41). L'une de ces conditions est que le texte ou au moins une partie du texte ait une fonction descriptive, une autre condition est que le mot sélectionné corresponde effectivement aux attentes engendrées par le type du texte ${ }^{42}$ (si elles existent) ; enfin, une condition est que la dénomination ne soit pas figurée. Les dénominations figurées servent à des buts bien précis de l'auteur. Des dénominations métonymiques qui vont à l'encontre des attentes et qui sont utilisées dans des contextes permettant des associations avec d'autres signes peuvent, par exemple, aider l'auteur à exprimer ses émotions ou à manipuler celles du destinataire. Par ailleurs, elles peuvent servir à empêcher une idée ou un problème de devenir thématique dans un contexte donné (cf. Zorman 2000b: 37 sq.).

A partir de telles observations, nous pouvons tenter d'identifier les facteurs qui déterminent le choix entre les synonymes au niveau textuel ${ }^{43}$. Le facteur primaire, d'après Coseriu (1971: 219), est l'objet désigné ${ }^{44}$ qui réduit le nombre de choix possibles. Ensuite, entrent en jeu la structure et la fonction textuelle ${ }^{45}$ ainsi que les propriétés formelles du mot. Une manière subtile d'opérer le choix entre des synonymes est d'établir des relations intertextuelles avec d'autres textes qui exigent l'emploi du même synonyme. Cependant, nous nous sommes plutôt focalisés ici sur le rôle des facteurs psychologiques et sociolinguistiques dans le choix du synonyme. Parmi les facteurs psychologiques, il y a les intentions, les sentiments et le pouvoir expressif de l'auteur. Quant aux facteurs sociolinguistiques, l'auteur ou le locuteur peut utiliser

\footnotetext{
39 Dans un texte dont le seul but devrait être de donner les informations objectives, les déviations intentionnelles par rapport à la norme représentent un mécanisme de manipulation en offrant la possibilité inattendue par le lecteur d'intégrer le savoir non linguistique dans la construction du sens ; voir Zorman (2000b: 36-38).

40 Outre les exemples déjà cités supra, nous pouvons y ranger aussi l'éditorial, le nouveau roman, l'essai, etc.

41 Voir note 40 supra.

42 Par exemple, dans des nouvelles, l'expression attendue pour la désignation des 'combats de rue' est boj et pas borba.

43 Voir à ce sujet Zorman (2000b: 39 sqq., 2005b).

${ }^{44}$ Sur le terme « objet », voir note 2 supra.

${ }^{45}$ Selon notre observation, ce sont la fonction descriptive, la fonction argumentative et la fonction persuasive qui peuvent exercer une influence décisive sur le choix synonymique.
} 
le choix entre les synonymes pour, en quelque sorte, s'identifier en tant que membre d'un groupe culturel et social donné. Il peut aussi s'en servir afin d'exprimer son attitude envers le destinataire. Le choix d'un synonyme au lieu d'une expression qu'un autre sujet communicant a employé pourrait indiquer un manque de solidarité sociale à l'égard de l'autre, ou bien une distance sociale entre les deux acteurs ${ }^{46}$.

Evidemment, cette description des fonctions potentielles du choix entre les synonymes n'est pas exhaustive. Elle démontre néanmoins que la synonymie abordée du point de vue de la pensée linguistique de Coseriu ne peut se réduire à la question de l'interchangeabilité des synonymes. En effet, des questions plus importantes se font jour comme celle du choix des synonymes fait par le locuteur et les raisons de ce choix. La théorie de Coseriu peut éclaircir les raisons de l'interchangeabilité des synonymes dans des contextes donnés, aussi bien que les différences de signification et du sens des synonymes dans les situations où le fait de remplacer un synonyme par un autre détruit ou transforme entièrement l'effet de l'énoncé.

\section{Bibliographie}

Apresjan, Jurij D. (1966): "Analyse distributionnelle des significations et champs sémantiques structurés". Languages 1: 44-74.

Apresjan, Jurij D. (1980): Semantyka leksykalna. Synonimiczne środki języka. Wrocław etc.: $\mathrm{ZNiO}$, Ossolineum.

Bierwisch, Manfred (1970): "Semantics". In: Lyons, John (éd.) (1979): New Horizons in Linguistics. Harmondsworth, Penguin: 166-185.

Carnap, Rudolf (1955): "Meaning and Synonymy in Natural Languages". Philosophical Studies 6: 33-47.

Coseriu, Eugenio (1970): Einführung in die strukturelle Betrachtung des Wortschatzes. Tübingen: Narr.

Coseriu, Eugenio ( $\left.{ }^{2} 1971\right)$ : Sprache. Strukturen und Funktionen. Tübingen: Narr

Coseriu, Eugenio (1973): Die Lage in der Linguistik. Innsbruck: Inst. für Sprachwiss. der Univ.

Coseriu, Eugenio (1975): Sprachtheorie und allgemeine Sprachwissenschaft. München: Fink.

Coseriu, Eugenio ( $\left.{ }^{2} 1985\right)$ : Textlinguistik. Darmstad: Wiss. Buschgesell.

Coseriu, Eugenio ( $\left.{ }^{2} 1992\right)$ : Einführung in die allgemeine Sprachwissenschaft. Tübingen: Francke.

Coseriu, Eugenio (2001): L'homme et son langage. Louvain-Paris-Sterling/Virgini: Peeters.

Coseriu, Eugenio (2003): Geschichte der Sprachphilosophie. Von den Anfängen bis Rousseau. Tübingen/Base: Francke.

Cruse, Alan D. (1986): Lexical semantics. Cambridge: Cambridge University Press.

De Cuypere, Ludovic/Willems, Klaas (2008): "Meaning and Reference in Aristotle's Concept of the Linguistic Sign". Foundations of Science 13/3-4: 307-324.

Ducháček, Otto (1964): "Différents types de synonymes". Orbis 13: 35-49.

Dupuy-Engelhardt, Hiltraud (1990): La saisie de l'audible : étude lexématique de l'allemand. Tübingen: Narr.

Eckert, Rainer (1968): "Zur Darstellung der Synonymie mit Hilfe der Distributionsmodelle und einige Schlußfolgerungen daraus für den russischen Sprachunterricht". Wissenschaftliche Zeitschrift der Karl-Marx-Universität. Gesellschafts- und Sprachwissenschaftliche Reihe 17: 129-132.

Filipec, Josef (1961): Česká synonyma z hlediska stylistiky a lexikologie. Prah: Nakl. Češkoslov. Akad. Věd.

Gabka, Kurt (1967): "Zur Abgrenzung lexikalischer, morphologischer und syntaktischer Synonymie". Zeitschrift für Slawistik 12: 727-734.

\footnotetext{
46 Sur cette fonction du choix synonymique, cf. Halliday et Hasan (1976: 274 sqq.).
} 
Gauger, Hans-Martin (1972): "Die Wörter und ihr Kontext. Ein Beitrag zu den Synonymen". Neue Rundschau 83: 432-450.

Halliday, Michael A. K./Hasan, Ruqiya (1976): Cohesion in English. London: Longman.

Harris, Roy (1973): Synonymy and Linguistic Analysis. Oxford: Blackwell.

Harris, Zellig S. (1970): Papers in Structural and Transformational Linguistics. Dordrecht: Reidel

Haßler, Gerda (à paraître): "La relation entre la philosophie du langage et la sémantique chez Coseriu". Disponible sur http://www.uni-potsdam.de/romanistik/hassler/pdf/Coseriu.pdf, consulté le 5 Janvier 2010.

Heringer, Hans Jürgen (1999): Das höchste der Gefühle. Empirische Studien zur distributiven Semantik. Tübingen: Stauffenburg.

Heringer, Hans Jürgen (s. a.): Distributionelle Semantik. Ein Arbeitspapier. Manuscrit non publié.

Heringer, Hans Jürgen (2010): "Semantischer Inspektor. Ein Computerprogramm für semantische Analyse". Disponible sur http://www.philhist.uniaugsburg.de/faecher/germanis/daf/neu/heringer/inspektor.php, consulté le 5 Janvier 2010.

Hjelmslev, Louis (1980): Prolegomena teoriji jezika. Zagreb: GZH.

Hundsnurscher, Franz (1992): Some Considerations on Semantic Descriptions. In: Stamenov, Maxim I. (éd.) (1992): Current Advances in Semantic Theory. Amsterdam/Philadelphia, Benjamins: 283-298.

Katz, Jerrold J./Fodor, Jerry A. (1963): "The Structure of a Semantic Theory." Language 39: $170-210$.

Lyons, John (1963): Structural Semantics. Oxford; BLackwell.

Lyons, John ( $\left.{ }^{4} 1975\right)$ : Einführung in die moderne Linguistik. München: Beck.

Lyons, John (1980): Semantik. Bd. I. München: Beck.

Lyons, John (1981): Language, Meaning and Context. London: Fontana.

Mel'čuk, Igor A./ Žolkovskij, Aleksandr K. (1970): "Towards a Functioning 'Meaning-Text' Model of Language". Linguistics 57: 10-47.

Missire, Régis (2006): "Sémantique des textes et modèle morphosémantique de l'interprétation". Texto! [en ligne], mars 2006, vol. XI, $\mathrm{n}^{\circ} 1$. Disponible sur http://www.revue-texto.net/Inedits/Missire/Missire_These.html, consulté le 3 Janvier 2010.

Mistrík, Jozef (1975): Žánre vecnej literatúry. Bratislav: SPN.

Muroi,Yoshiyuki (1998): "Methodische Überlegungen zu einer korpusbasierten Semantikanalyse - Probleme und Möglichkeiten der Affinität". Disponible sur http://www.f.waseda.jp/ymuroi/pub/AFFIN.pdf, consulté le 2 Janvier 2010.

Murphy, M. Lynne (2003): Semantic Relations and the Lexicon: Antonymy, Synonymy, and Other Paradigms. Cambridge: Cambridge University Press.

Nida, Eugene A. (1975): Componential Analysis of Meaning. The Hague: Mouton

Putnam, Hilary (1954): "Synonymity and the Analysis of Belief Sentences". Analysis 14: 114-122.

Rastier, François (1989): "Sens et textualité". Disponible sur http://www.revuetexto.net/Parutions/Sens-et-textualite/Rastier_sens_et_textualite.html, consulté le 2 Janvier 2010.

Rastier, François (1990): "La triade sémiotique, le trivium et la sémantique linguistique". $\begin{array}{lllll}\text { Nouveaux actes sémiotiques } & 9 . & \text { Disponible } & \text { sur }\end{array}$ http://revues.unilim.fr/nas/document.php?id=2641, consulté le 2 Janvier 2010.

Rey-Debove Josette (1997): "La synonymie ou les échanges de signes comme fondement de la sémantique". Langages 31: 91-104. Disponible sur http://www.persee.fr/web/revues/home/prescript/article/lgge_0458-

726X_1997_num_31_128_2135, consulté le 24 Janvier 2012.

Quine, Willard Van Orman (1960): Word and Object. Cambridge, Mass.: MIT Press. 
Schirn, Matthias (1975): Identität und Synonymie. Stuttgart/Bad Cannstatt: FrommannHolzboog.

SSKJ = Slovar slovenskega knjižnega jezika I-V (1970-1991). Ljubljan: DZS.

Ullmann, Stephen (1967): Grundzüge der Semantik. Berlin: de Gruyter.

Zorman, M. (1998a) "Stilno nevtralni izrazi v funkciji vplivanja na vrednotenje". In: Štrukelj, I. (éd.) (1998): Jezik za danes in jutri. Ljubljana, Društvo za uporabno jezikoslovje Slovenije: $216-222$.

Zorman, Marina (1998b): "Zur Struktur und Funktion von Synonymen." Linguistica 38/2: $3-62$.

Zorman, Marina (2000a): O sinonimiji. Ljubljana: Znanstveni Inštitut Filozofske Fak.

Zorman, Marina (2000b): "Izbira med sinonimi v besedilu: o funkcijah in dejavnikih."In: Razprave Slovenske akademije znanosti in umetnosti, Razred za filološke in literarne vede: 29-44. ( =Dissertationes Academiae scientiarium et Artium Slovenica, Clasis II: Philologia et litterae 17).

Zorman, Marina (2005a): Distribution und Bedeutung der Synonyme. In: Ehrhardt, Horst/Zorman, Marina (éds.) (2005): Semantische Probleme des Slowenischen und des Deutschen. Frankfurt am Main etc., Lang: 315-335.

Zorman, Marina (2005b): "Zvrstnost v retoriki praktičnega sporazumevanja." Slovenski jezik - Slovene Linguistic Studies 5: 85-97.

Zvegincev, Vladimir A. (1963): Zamečanija o leksičeskoj sinonimii. In: (s. n.): Voprosy teorii $i$ istorii jazyka. Sbornik $v$ čest' prof. B. A. Larina. Leningrad, Izdat. Pjatigorskogo Gosudarstvennogo Pedagogičeskogo Inst. Inostrannych Jazyko: 127-142. 